\title{
Homeostatic Process
}

National Cancer Institute

\section{Source}

National Cancer Institute. Homeostatic Process. NCI Thesaurus. Code C25989.

The body's maintenance of a consistent internal environment within a narrow range of variation. It is important to the continuation of life. 